DOG

Deutsche Ophthalmologische Gesellschaft

Platenstr. 1

80336 München

Tel.: +49 (0)895505 768-15

Fax: +49 (0)895505 768-11

Geschaeftsstelle@dog.org

www.dog.org

\title{
Ausschreibung von Preisen und Forschungsförderungen 2016
}

\begin{abstract}
Bitte senden Sie Ihre Bewerbung - sofern nachfolgend nicht anders angegeben - per E-Mail an die Geschäftsstelle der DOG (geschaeftsstelle@dog.org) Deadline: 1. Mai 2016

Weitere wichtige Informationen zu den ausgeschriebenen Förderungen finden Sie auf der Webseite der DOG (www.dog.org) im Bereich Förderungen/aktuelle Ausschreibungen.

Es können nur Bewerbungen/Arbeiten berücksichtigt werden, die nicht bereits für andere Ausschreibungen von Preisen und Forschungsförderungen der DOG oder Dritter eingereicht bzw. ausgezeichnet wurden.
\end{abstract}

\section{Forschungsförderungen}

\section{- Forschungsförderung}

Tropenophthalmologie der DOG - Die DOG fördert im Jahr 2016 Forschungsvorhaben im Bereich Tropenophthalmologie bis zu einer Summe von 5.000 Euro.

\section{Kurzzeitdozenturen}

- Auslands-Kurzzeitdozenturen der DOG Die DOG fördert im Jahr 2016 drei Kurzzeitdozenturen für deutsche Professoren und Dozenten an Universitäten in Afrika, Asien und Lateinamerika mit bis zu je 3.000 Euro.

\section{Preise}

- Elfriede-Aulhorn-Preis gestiftet von der Gesellschaft zur Förderung der neuro-ophthalmologischen Forschung e. V. und verliehen für besonders wertvolle Arbeiten im Bereich der Physiologie und Pathophysiologie des Sehens sowie der Neuroophthalmologie. Die Dotierung beträgt 4.000 Euro. Bewerbungen sind bis zum 1. Mai 2016 an Herrn Prof. Dr. E. Zrenner, Univ.-Augenklinik Tübingen zu richten.

- Glaukomforschungspreis ausgeschrieben durch die DOG, gestiftet von der Santen GmbH, München, zur Anerkennung herausragender Arbeiten auf dem Gebiet der experimentellen und klinischen Glaukomatologie. Die Dotierung beträgt 5.000 Euro.

\section{- Leonhard-Klein-Preis} ausgeschrieben und gestiftet durch die LeonhardKlein-Stiftung für Arbeiten, die sich mit ophthalmochirurgischer Grundlagenforschung zur Verbesserung von Operationsverfahren, klinischen Studien zu Operationsver- fahren sowie Wirkungen von chirurgischen Eingriffen auf die Regulationsvorgänge des Auges befassen. Die Dotierung beträgt 15.000 Euro. Bitte senden Sie Ihre Vorschläge bis zum 31. März 2016 in elektronischer Form als pdfDatei an den Stifterverband für die Deutsche Wissenschaft, doris.zirkler@stifterverband.de z. Hd. Frau Doris Zirkler, Postfach 1644 60, 45224 Essen. Bitte beachten Sie, dass Eigenbewerbungen für diese Auszeichnung nicht möglich sind.

- Retina-Förderpreis - ausgeschrieben durch die DOG, gestiftet von der Novartis Pharma GmbH, Nürnberg, zur Förderung innovativer Entwicklungen und therapeutischer Ansätze auf dem Gebiet von Netzhauterkrankungen. Die Dotierung beträgt 5.000 Euro.

- Wissenschaftspreis Trockenes Auge und Blepharitis/MGD - ausgeschrieben durch die DOG, gestiftet von der Optima Pharmazeutische $\mathrm{GmbH}$, Moosburg, zur Auszeichnung herausragender klinischer und grundlagenwissenschaftlicher Arbeiten zum Trockenen Auge und Blepharitis/Meibom-Drüsen Dysfunktion (MGD). Der Preis wird in zwei Kategorien (klinische bzw. grundlagenwissenschaftliche Arbeit) mit einer Dotierung von jeweils 3.000 Euro vergeben.

- Wissenschaftspreis der Stiftung Auge - ausgeschrieben und gestiftet von der Stiftung Auge zur Förderung für wissenschaftlich hervorragende Originalveröffentlichungen auf dem Gebiet der Augenheilkunde. Die Dotierung beträgt 2.500 Euro. Bewerben können sich Nachwuchswissenschaftler/innen an Universitätskliniken oder Universitätsinstituten sowie wissenschaftlich orientierte, praktizierende Augenärzte/ Augenärztinnen. Die Bewerbungsarbeiten sind online zusammen mit einem Curriculum vitae der Bewerberin/des Bewerbers sowie einer Aufstellung und Beschreibung von bereits erhaltenen Förderungen und Auszeichnungen einzureichen bei der Geschäftsstelle der Stiftung Auge (info@stiftung-auge.de). Frist für die Abgabe der Bewerbungen ist der 1. Mai 2016. 